\title{
ESPÉCIES DE Passiflora L. (PASSIFLORACEAE) PUBLICADAS E DESCRITAS NOS ÚLTIMOS 55 ANOS (1950 - 2005) NA AMÉRICA DO SUL E PRINCIPAIS PUBLICAÇÕES BRASILEIRAS.
}

\author{
Species of Passiflora L (Passifloraceae) published and described \\ during the last 55 years (1950-2005) in South America and in \\ the main brazilian publications
}

Armando Carlos Cervi

\section{Resumo}

Este trabalho é o resultado das espécies descritas do gênero Passiflora L. dos últimos 55 anos (1950 - 2005) para a América do Sul. Além das espécies descritas neste período foi realizado o levantamento das principais publicações sistemáticas para o gênero Passiflora no Brasil.

Palavras-chave: Passiflora L.; Espécies descritas de Passiflora; Passiflora na América do Sul; Passifloraceae na América do Sul.

\section{Abstract}

This work is a listing of the species of Passiflora L. genus described during the last 55 years (1950-2005) for South America. Apart from the species described during this period, the main Brazilian publications of Systematics for Passiflora genus are quated.

Keywords: Passiflora L; Passiflora species described; South America Passiflora; South America Passifloraceae.

1 Departamento de Botânica da Universidade Federal do Paraná.

Pesquisador Bolsista do CNPq. E-mail: accervi@ufpr.br

Caixa Postal 19041. CEP 81531-980 Curitiba. Paraná. Brasil. 


\section{Introdução}

A família Passifloraceae está dividida em duas tribos - Paropsieae e Passiflorieae. Essa última está representada no continente latino-americano por quatro gêneros: Ancistrothyrsus Harms, Dilkea Mast., Mitostemma Mast. e Passiflora L. Dentre estes, destaca-se o gênero Passiflora L. O gênero Passiflora, atualmente, é estimado em aproximadamente 520 espécies para o mundo.

O estudo da família Passifloraceae iniciouse com Linnaeus, em 1753. Entretanto, a literatura essencial se configuraria mediante a relevante contribuição de pesquisadores brasileiros e estrangeiros, nos séculos XIX e XX, entre os quais é de justiça reconhecer o mérito de J.M.C. Velloso (1827 (1831)), A.P. De Candolle (1828), M.T. Masters (1871, 1872), J. Barbosa Rodrigues (1891), H. Harms (1893, 1922, 1925, 1929), A. Usteri (1911) e E. P. Killip (1924, 1938, 1960) e A. L. Uribe-Uribe (1955).

A recentividade das pesquisas taxonômicas, relacionadas à família Passifloraceae para a América do Sul, dadas a conhecer no período 1950 a 2005 e realizadas por um número igualmente restrito de especialistas, demanda um confronto de resultados de modo a proporcionar matéria para uma revisão crítica do repertório de conhecimento a consagrar. A esse intuito deve servir o presente trabalho.

\section{Material e Métodos}

A presente revisão está pautada em consultas a obras de Botânica sistemática e a estudos publicados e/ou divulgados em eventos científicos no período 1950-2005. O material analisado pertence a acervos de bibliotecas nacionais e estrangeiras e ao acervo particular do Autor.

\section{Resultados}

Espécies descritas para a América do Sul

1954. P. mariquitensis Mutis ex L. Uribe - Colombia - Mutisia 21:1. f. 1.

1954. P. crispolanata L. Uribe - Colômbia - Mutisia. 21:5. f. 2.

1955. P. azeroana L. Uribe - Colômbia - Fl. Real. Exped. Bot. Nuevo Reino Granada. 27:35. f. 11.
1957. P. arbelaezii L. Uribe - Colômbia - Caldasia, 7(35):335. f. 1.

1958. P. albicans L Uribe - Colômbia - Caldasia. 8:27. f. 1. = P.uribei L Escobar -Sin. válido - Fl. Colombia. 10:64

1960. P. cuatreca sa sii Killip - Colômbia - Contr. U. S. Nat. Herb. 35(1):7. f. 2.

1960. P. killipiana Cuatrecasas - Colombia - Contr. U. S. Nat. Herb. 35(1)17. f. 10.

1960. P. macropoda Killip - Bolívia - Contr. U. S. Nat. Herb. 35(1):14. f. 8.

1960. P. danielii Killip - Colômbia - Contr. U. S. Nat. Herb. 35(1):19. f. 11

1960. P. ursina Killip e Cuatrecasas - Colômbia Contr. U. S. Nat. Herb. 35(1):2. f. 1.

1960. P. raimondii Killip - Peru - Contr. U. S. Nat. Herb. 35(1):10. f. 1.

1960. P. loxensis Killip e Cuatrecasas - Equador Contr. U. S. Nat. Herb. 35(1):12. f. 7.

1960. P. boyacana Killip - Colômbia - Contr. U. S. Nat. Herb. 35(1):9. f. 4.

1960. P. zamorana Killip - Equador - Contr. U. S. Nat. Herb. 35(1):11.

1966. P. imbeana Sacco - Brasil, RJ - Sellowia 18: 41.

1966. P. edmundoi Sacco - Brasil, BA. e MG. Sellowia 18: 41-47

1966. P. emiliae Sacco - Brasil, MT - Boletim do Museu Nacional. 32:1. Atualmente é sinônimo de P. ambigua Hemsl.

1966. P. amicorum J.J. Wurdack - Venezuela - Bol. Socied. Venez. Cienc. Nat. 26:429. f. 5.

1967. P. tholozanii Sacco -Brasil, AM. - Anais do XV Congresso Nacional de Botânica. Soc. Bot. do Brasil. Vol.1: 151.

1967. P. araujoi Sacco - Brasil, PA. e MA. - Anais do XV Congresso Nacional de Botanica. Soc. Bot. do Brasil. Vol.1: 251.

1967. P. margaritae Sacco Brasil, ES. - Sellowia 19: 59.

1967.P.bomareifolia Steyerm. \& Maguire Venezuela - Mem. New York Bot. Gard. 17(1):455. f. 1.

1968. P. trintae Sacco - Brasil, MG. e BA. - Sellowia 20: 21.

1968. P. muriensis J. Steyerm. - Venezuela - Acta Bot. Venez. 3(1-4):188. f. 13.

1971. Passiflora cerradense Sacco - Brasil, MT, GO. e DF. - Ed. Edgard Blücher Ltda e Ed. USP. Vol.1:212. 1973. P. pilosicorona Sacco - Bolívia - Bradea 1(33): 349. 
1973. P. castellanosii Sacco - Brasil, CE. - Bradea 1(32): 345.

1974. P. harlingii Holm-Niels. - Equador - Botaniska Notisier, 127:339. f. 1.

1974. P. sparrei Holm-Niels. - Equador - Botaniska Notisier, 127:344. f. 3.

1974. P. caucaense Holm-Niels. - Equador Botaniska Notisier, 127:348. f. 4, 5.

1980. P. catarinensis Sacco - Brasil, SC. - Fl. Ilustr. Catarinensis. Fasc. Pass. p.60.

1980. P. reitzii Sacco -Brasil, SC. - Fl. Ilustr. Catarinensis. Fasc. Pass. p. 73.

1980. P. caudata A Gentry - Colômbia - Phytologia 47(2):103.

1981. P. involucrata A. Gentry - Colômbia - Plant Systematics and Evolution.137:101. f. 2.

1981. P. amethystina var bolosii Cervi - Brasil, PR. - Univ.de Barc. Centre de Publicacions Vol.1: 16. Sinonimizada para P. loefgrenii Vitta.

1984. P. crenata Feuillet \& Cremers - Guiana Francesa - Proc. Kon. Ned. Akad. Wetensch. C 87(4):378. f. 1.

1984. P. plumosa Feuillet \& Cremers - Guiana, Suriname e Guiana Francesa - Proc. Kon. Ned. Akad. Wentensch. C 87(4):381. f. 2.

1986. P. rufostipulata Feuillet - Guiana Francesa Candolea. 41:173. f. 1.

1986. P. fanchonae Feuillet - Guiana Francesa Candolea 41:175. f. 2.

1986. P. colombiana L. Escobar - Colômbia Systematic Botany. 11(1):95. f. 5.

1986. P. runa L. Escobar - Peru - Systematic Botany. 11(1):90. f. 3.

1986. P. huamachucoensis L. Escobar - Peru Systematic Botany. 11(1):88. f. 1.

1887. P. pergrandis Holm-Niels. \& Lawesson - Equador - Ann. Miss. Bot. Gard. 74:501, f. 4.

1987. P. deltoifolia Holm-Niels.\& Lawesson - Equador - Ann. Miss. Bot. Gard. 74:501, f. 3.

1987. P. montana Holm-Niels. \& Lawesson - Equador - Ann. Miss. Bot. Gard. 74:499, f..2.

1987. P. palequensis Holm-Niels.\& Lawesson - Colômbia - Ann. Miss. Bot. Gard. 74: 497. f. 1.

1987. P. sanctae-barbarae Holm-Niels. \& P. Jorgensen

- Equador - Nordic J. Bot. 7(2): 132, f. 4.

1987. P. discophora P. Jorgensen \& Lawesson - Equador - Nordic J. Bot. 7(2):127. f. 1.

1987. P. hirtiflora P. Jorgensen \& Holm-Niels. Equador - Nordic J. Bot. 7(2):132, f. 3 a,b,c.

1987. P. monadelpha P. Jorgensen \& Holm-Niels. Equador - Nordic J. Bot. 7(2): 129, f. 2.
1987. P. subpurpurea P. Jorgensen \& Holm-Niels. Equador - Nordic J. Bot. 7(2): 131, f. 3 d,e,f,g.

1988. P. linearistipula L Escobar - Colômbia - Fl. Colombia. 10:39. f. 11

1988. P. pacifica L Escobar - Colômbia - Mutisia. 71:1. f. $1,2$.

1988. P. jardinensis L Escobar - Colômbia - Mutisia. 71:4. f. 3.

1988. P. tenerifensis L Escobar - Colômbia - Mutisia. 71:4. f. 4.

1988. P. uribei L. Escobar - Colômbia - Fl. Colombia. 10:64. f. 17.

1989. P. amazonica L. Escobar - Peru - Ann. Miss. Bot. Gard. 76:876. f. 1.

1989. P. pascoensis L. Escobar - Peru - Ann. Miss. Bot. Gard. 76:880. f. 3.

1989. P. sierrae L Escobar - Colômbia - Ann. Miss.

Bot. Gard. 76:884. f. 4.

1989. P. chlorina L. Escobar - Brasil, MG. Phytologia 67(2):132.

1989. P. caatingae L Escobar - Brasil, BA. - Ann. Miss. Bot. Gard.76:880. f. 2. Atualmente é sinônimo de P. trintae Sacco

1989. P. solomonii L Escobar - Bolívia - Ann. Miss. Bot. Gard. 76:884. f. 5.

1989. P. fernandezii L. Escobar - Bolívia Phytologia 66(1):80.

1989. P. viridescens L. Escobar - Peru - Phytologia 66(1):81.

1990. P. araguensis L. Escobar - Venezuela Phytologia. 669:364

1990. P. magnifica L. Escobar - Colômbia Phytologia 69(5):365. f. 1.

1992. P. faneyi Pessoa \& Cervi - Brasil, RJ. Candollea 47: 631. f. 1.

1992. P. escobariana MacDougal - Colômbia Novon. 2(4):365. f. 6.

1992. P. brachyantha L. Escobar - Equador - Novon 2(3):198. f. 1.

1994. P. saccoi Cervi - Brasil, MG. - Brittonia 46: 144. f. 1.

1994. P. hatschbachii Cervi - Brasil, MG. Fontqueria 40: 45. f. 1.

1994. P. callistemma L Escobar - Colômbia - Syst. Bot. 19(2):205.

1994. P. kawensis Feuillet - Guiana Francesa Novon 4(3):236. f. 1

1994. P. exura Feuillet - Guiana, Suriname, Guiana Francesa - Novon. 4(3):238. f. 2.

1994. P. kawensis Feuillet - Guiana, Suriname, Guiana Francesa -Novon. 4(3):236. f. 1. 
1996. P. quadrifaria J. Vanderplank. - Brasil, AM. (Jari). - Curtis's Botanical Magazine 13: 63.

1996. P. trialata Feuillet \& MacDougal - Guiana, Suriname, Guiana Francesa - Novon. 6(4). 351. f. 1 a,b; 2 a,b.

1996. P. linda Panero - Equador - Brittonia. 48(2): 192. f.1.

1997. P. loefgrenii Vitta - Brasil, SP - Novon 7(2): 210.

1997. P. cerasina H. Annonay \& Feuillet - Guiana Francesa - Sida 17(3):551.

1997. P. chrysosepala M. Schwerdtfeger - Equador - Haussknechtia, Mitt. Thüring Bot. Ges. 6:41

1997. P. jatunsachensis Schwerdtfeger - Equador Hausknechtia (Jena) 6:43

1997. P. trochlearis P. Jorgensen - Equador - Novon 7(4):379, f. 1.

1997. P. luzmarina P. Jorgensen - Equador - Novon 7(4):385, f. 2.

1997. P. smilacifolia MacDougal - Equador - Novon 7(4):382.

1997. P. ulmeri M. Schwerdtfeger - Equador Haussknechtia, Mitt. Thüring Bot. Ges. 6:46

1998. P. telesiphe S.Knapp \& J. Mallet - Equador Novon 8(2):162. f. 1a-c, 2.

1999. P. formosa T. Ulmer - Colômbia - Edinburgh J. Bot. 56(2):196. f. 1.

2000. P. chocoensis G. Gerlach \& T. Ulmer Colombia - Caldasia 22(2):231. f. 1, 2.

2000. P. weigendii T. Ulmer \& M. Schwerdtfeger Peru - Nordic J. Bot. 20(1):47.

2001. P. sargasteguii Skrabal \& Weigend - Peru Harvard Pap. Bot. 6(1):321. f. 11 a-c, 15 g,h.

2001. P. tarminiana Coppens \& Barney - Colombia - Novon 11(1):8. f. 1.

2001. P. tina Boender \& T. Ulmer - Equador Dendtnera 7:6 f. 1-3.

2002. P. cordistipula Cervi - Brasil, AM. - Brittonia 54: 54. f. 1.

2002. P. ascidia Feuillet - Venezuela - Brittonia 54(1):20. f. 1 a-d

2002. P. balbis Feuillet - Guiana Francesa - Brittonia 54(1):20. f. 2 a-f

2002. P. inca P. Jorg. - Peru - Novon 14(1)79. f. 1,2. 2002. P. markiana A. K. Hansen - Equador Lundellia 5:44. fig. 1.

2002. P. saulensis Feuillet - Guiana Francesa Brittonia 54(1): 24. f. 3 a-c

2003. P. urubicensis Cervi - Brasil, SC. - Sellowia 53-55: 9. f. 1

2003. P. vescoi D. Rignon \& L. Rignon - Guiana Francesa - Adansonia 22(2)219.
2004. P. contracta Vitta - Brasil, ES.e PE. - Brittonia 56: 89.

2004. P. carnosisepala P. Jorg. - Equador - Nordic J. Bot. 23(1):11. f. 14.

2004. P. parvipetala P. Jorg. - Equador - Nordic J.

Bot. 23(1):15. f.1.

2004. P. phellos Feuillet - Venezuela - Novon 14(3):285. f. 1.

2005. P. mucugeana T.S.Nunes \& L.P.Queiroz - Brasil, BA. - Submetido a Acta Botanica Brasilica.

2005. P. boticarioana Cervi - Brasil, MG. - Submetido a Brittonia.

\section{Principais trabalhos de sistemática e Tax onomia da família Passiflora ceae publicados no Brasil, a lém dos já citados nas espécies descritas.}

1. Hoehne, F.C.; Kuhlmann, J.C. (org.). Índice bibliográfico e numérico das plantas colhidas pela comissão Rondon ou Comm. Linh. Telegr. Mato Grosso ao Amazonas de 1908 até 1923. Passifloraceae. São Paulo: Secretaria de Agricultura 1951; 291-294.

2. Sacco J. da C. Passifloraceae. Flora ilustrada do Rio Grande do Sul. Bol. Inst. Cienc. Nat., UFRGS, Porto Alegre, 1962; 12(4): 7-29, fig. 1-13.

3. Semir J.; Brown Jr., K.S. Maracujá: a flor da paixão. Ver. Geogr. Univ., 1975; 5(2): 40-47.

4. Valente M. da C. Levantamento dos "tipos" das espécies de Passifloraceae e Rhizophoraceae do herbário do Jardim Botânico do Rio de Janeiro. Arq. Jard. Bot., Rio de Janeiro, 1977; 20: 21-25, fig. 1-2.

5. Santos, E.; Costa N.L.M.; Guimarães, A.M.C.D. Os Typus das plantas vasculares do herbário do Museu Nacional III Bol Mus Nac, Bot, Rio de Janeiro 1978; 49: 1-50, fig. 1-55.

6. Sacco J. da C. Passifloraceae. In: Reitz, R. (ed.). Flora ilustrada catarinense. Itajaí : Herbário Barbosa Rodrigues 1980; 130.

7. Cervi, A.C. Revisión del genero Passiflora L. (Passifloraceae) del estado do Paraná, Brasil. Bar- 
celona. Tesis - (Doctorado). Universitat de Barcelona Resumen 1982; 26p.

8. Cervi, A.C.; Bidá, A. Redescrição de Passiflora setulosa Killip. Collect. Bot, Barcelona 1983; 14:247-251.

9. Cervi, A.C. Passifloraceae. In: Rizzo J.A. (coord.). Flora do Estado de Goiás. Goiânia : UFG, 1986; 7:1-45. (Coleção Rizzo).

10. Cervi, A.C. Estudo sobre Passifloraceae I. Ocorrência de Passiflora foetida var. nigelliflora (Hooker) Masters e Passiflora warmingii Masters no Paraná, Brasil. Acta Biol. Paranaensis., Curitiba 1990; 19(1,2,3,4):159-169, fig. 1-3.

11. Cervi, A.C. Passifloraceae. In: Barros, F, et al. (eds.) Flora fanerogâmica de Ilha do Cardoso. São Paulo : Instituto de Botânica, 1991; 1: 153.

12. Cervi, A.C. Passifloraceae. In: Barros, F. et al. (eds.). Flora fanerogâmica de Ilha do Cardoso. São Paulo : Instituto de Botânica, 1992; 3:11-20, fig.1-4.

13. Cervi, A,C. Ocorrência de Passiflora miersii Masters para o estado do Paraná e outras regiões brasileiras. Acta Biol. Paranaensis, Curitiba 1994; 23(1,2,3,4):73-78, fig. 1-2.

14. Pessoa, S.V.A. Passifloraceae. In: Lima, M.P.M.; Guedes-Bruni, R.R. (org.). Reserva Ecológica de Macaé de Cima, Nova Friburgo, RJ. : aspectos florísticos das espécies vasculares. Rio de Janeiro : Jardim Botânico do Rio de Janeiro, 1994; 1:315322, fig. 1.

15. Cervi, A.C. De Passifloris brasiliensibus notulae quaedam. Fontqueria, Madrid, 1995; 42:87-90.

16. Vitta, F.B. Passifloraceae. In: Stannard, B.L (ed.). Flora of the Picos das Almas: Chapada Diamantina, Bahia, Brazil. Kew : Royal Botanic Gardens 1995; 526-528.

17. Cervi, A.C. Passifloraceae da região de Carangola - Minas Gerais, Brasil. Pabstia, Carangola, 1996; 7:1-32.

18. Cervi, A.C. Passifloraceae do Brasil: estudo do gênero Passiflora L. subgênero Passiflora. Fontqueria, Madrid, 1997; 45:1-92, figs. 1-12.
19. Cervi, A.C. Passifloraceae. In: Dubs, B. Prodromus Florae Matogrossensis . Betrona :Verlag, 1998; Part. I., 1: 235-237; Part. II, v.1, p.420-421.

20. Cervi, A.C. Passifloraceae. In: Dubs, B. Prodromus Florae Matogrossensis. Betrona :Verlag, 1998; Part. II, 1:420-421.

21. Bernacci, L.C.; Vitta, F.A. Flora fanerogâmica da reserva do parque estadual das Fontes do Ipiranga, São Paulo, Brasil. Hoehnea, São Paulo, 1999; 26(2):135-147.

22. Hopkins, M.J.G.; Souza, M.A.D. In. Ribeiro, J.E.LS. et al. Flora da reserva Duke: guia de identificação das plantas vasculares de uma floresta de terra-firme da Amazônia Central. Manaus : INPADFID, 1999; 299-306.

23. Cervi, A.C. Estudo das Passifloraceae Brasileiras: o subgênero Dysosmioides Killip do gênero Passiflora para o Brasil. Estad. Biol. (Curitiba), 2000; 45:91-115.

24. Bernacci, L.C. Notas sobre Passiflora ischnoclada Harms (Passifloraceae). Acta Bot. Brás., Porto Alegre, 2001; 15(2): 197-199.

25. Mondin, C.A. Passiflora organensis Gardner (Passifloraceae), primeira citação de ocorrência para o Rio Grande do Sul. Pesquisas, Bot., São Leopoldo, 2001; 51: 147-150, fig. 1-2.

26. Nunes, T.S.; Queiroz, L.P. A família Passifloraceae na Chapada Diamantina, Bahia, Brasil. Sitientibus, Cienc. Biol., Feira de Santana, 2001; 1(1): 33-46, fig. 1-6.

27. Nunes, T.S.; Zappi, D.C.; Queiroz, L.P. Lectotypification of two species of Passiflora (Passifloraceae). Kew Bull, London, 2001; 56: 245-246.

28. Bernacci, LC. Passifloraceae. In: Wanderley, M.G.L; Shepherd, G.J.; Melhem, T.S.; Giulietti, A.M.; Kirizawa, M. (eds.). Flora Fanerogâmica de São Paulo. São Paulo : Instituto de Botânica, 2003; 3: 247-274, figs 1-2.

29. Cervi, A.C.; Dunaiski Jr., A. Passifloraceae do Brasil: estudo do gênero Passiflora L subgênero 
Distephana (Juss.) Killip. Estud. Biol. (Curitiba), 2004; 26(.55): 45-67, fig. 1-7.

30. Milward-de-Azevedo, M.A.; Baumgratz, J.F.A. Passiflora L. subgênero Decaloba (DC.) Rchb. (Passifloraceae) na região Sudeste do Brasil. Rodriguésia, Rio de Janeiro 2004; 55(85): 1-54, fig. 1-19.

31. Milward-de-Azevedo, M.A.; Valente, M.C. Passifloraceae da mata de encosta do Jardim Botânico do Rio de Janeiro, Rio de Janeiro, RJ Arq. Mus. Nac., Rio de Janeiro 2004; 62(4): 367-374, fig. 1-3.

32. Milward-de-Azevedo, M.A.; Gonçalves-Esteves, V.; Baumgratz, J.F.A. Palinotaxonomia das espécies de Passiflora L. subg. Decaloba (DC.) Rchb. (Passifloraceae) no Sudeste do Brasil. Ver. Bras. Bot., Brasília, 2004; 27(4): 655-665, fig. 1-59.
33. Vitta, F.A.; Bernacci, LC. A new species of Passiflora in section Tetrastylis (Passifloraceae) and two overlooked species of Passiflora from Brazil. Brittonia, Bronx, 2004; 56(1): 89-95.

34. Bernacci, L.C.; Maletti, LM.M.; Soares-Scott, M.D.; Passos, I.R.S. Espécies de maracujá: caracterização e conservação da biodiversidade. In: Faleiro, F.G.; Junqueira, N.T.V.; Braga, M.F. (eds.). Maracujá: germoplasma e melhoramento genético. Planaltina - DF. Embrapa - Cerrados, 2005; 559586. figs. 1-5.
Recebido em / Received: July 4, 2005. Revisado em / Revised: September 20,2005. Aceito em / Accepted: October 31, 2005. 